\title{
Measure-preserving homeomorphisms of noncompact manifolds and mass flow toward ends
}

by

\author{
Tatsuhiko Yagasaki (Kyoto)
}

\begin{abstract}
Suppose $M$ is a noncompact connected $n$-manifold and $\omega$ is a good Radon measure of $M$ with $\omega(\partial M)=0$. Let $\mathcal{H}(M, \omega)$ denote the group of $\omega$-preserving homeomorphisms of $M$ equipped with the compact-open topology, and $\mathcal{H}_{E}(M, \omega)$ the subgroup consisting of all $h \in \mathcal{H}(M, \omega)$ which fix the ends of $M$. S. R. Alpern and V. S. Prasad introduced the topological vector space $\mathcal{S}(M, \omega)$ of end charges of $M$ and the end charge homomorphism $c^{\omega}: \mathcal{H}_{E}(M, \omega) \rightarrow \mathcal{S}(M, \omega)$, which measures for each $h \in \mathcal{H}_{E}(M, \omega)$ the mass flow toward ends induced by $h$. We show that the map $c^{\omega}$ has a continuous section. This induces the factorization $\mathcal{H}_{E}(M, \omega) \cong \operatorname{Ker} c^{\omega} \times \mathcal{S}(M, \omega)$ and implies that $\operatorname{Ker} c^{\omega}$ is a strong deformation retract of $\mathcal{H}_{E}(M, \omega)$.
\end{abstract}

1. Introduction. This article is a continuation of the study of groups of measure-preserving homeomorphisms of noncompact topological manifolds $[2,3,4,8]$. Suppose $M$ is a noncompact connected $n$-manifold and $\omega$ is a good Radon measure of $M$ with $\omega(\partial M)=0$. Let $\mathcal{H}(M, \omega)$ denote the group of $\omega$-preserving homeomorphisms of $M$ equipped with the compact-open topology. In the study of this group, the space $E_{M}$ of ends of $M$ plays a significant role. Let $E_{M}^{\omega}$ denote the open subset of $E_{M}$ consisting of $\omega$ finite ends of $M$ and let $\mathcal{H}_{E_{M}}(M, \omega)$ denote the subgroup consisting of all $h \in \mathcal{H}(M, \omega)$ which fix the ends of $M$.

In [1] S. R. Alpern and V. S. Prasad introduced the end charge homomorphism

$$
c^{\omega}: \mathcal{H}_{E_{M}}(M, \omega) \rightarrow \mathcal{S}(M, \omega)
$$

An end charge of $M$ is a finitely additive signed measure on the algebra of clopen subsets of $E_{M}$. Let $\mathcal{S}\left(E_{M}\right)$ denote the topological linear space of all end charges of $M$ with the weak topology and let $\mathcal{S}(M, \omega)$ denote the linear subspace of $\mathcal{S}\left(E_{M}\right)$ consisting of end charges $c$ of $M$ with $c\left(E_{M}\right)=0$ and

2000 Mathematics Subject Classification: 57S05, 58C35.

Key words and phrases: group of measure-preserving homeomorphisms, mass flow, end charge, $\sigma$-compact manifold. 
$\left.c\right|_{E_{M}^{\omega}}=0$. For each $h \in \mathcal{H}_{E_{M}}(M, \omega)$ an end charge $c_{h}^{\omega} \in \mathcal{S}(M, \omega)$ is defined by

$$
c_{h}^{\omega}\left(E_{C}\right)=\omega(C-h(C))-\omega(h(C)-C),
$$

where $C$ is any Borel subset of $M$ such that $\operatorname{Fr} C$ is compact and $E_{C} \subset E_{M}$ is the set of ends of $C$. This quantity is the total $\omega$-volume (or mass) transferred by $h$ into $C$ and into $E_{C}$ at the last. Hence, the end charge $c_{h}^{\omega}$ measures mass flow toward ends induced by $h$.

In [3] R. Berlanga showed that the group $\mathcal{H}(M, \omega)$ is a strong deformation retract of the group $\mathcal{H}(M, \omega$-e-reg $)$ consisting of $\omega$-end-regular homeomorphisms of $M$. The group $\mathcal{H}(M, \omega$-e-reg) acts continuously on the space $\mathcal{M}_{\mathrm{g}}^{\partial}(M, \omega \text {-e-reg })_{\mathrm{ew}}^{*}$ of good Radon measures $\mu$ on $M$ such that $\mu(M)=\omega(M)$, $E_{M}^{\mu}=E_{M}^{\omega}$ and $\mu$ and $\omega$ have the same null sets, equipped with the finiteend weak topology. He showed that the orbit map $\pi: \mathcal{H}(M, \omega$-e-reg $) \rightarrow$ $\mathcal{M}_{\mathrm{g}}^{\partial}(M, \omega \text {-e-reg })_{\text {ew }}: h \mapsto h_{*} \omega$ has a continuous section. This section induces the factorization $\mathcal{H}(M, \omega$-e-reg $) \cong \mathcal{H}(M, \omega) \times \mathcal{M}_{\mathrm{g}}^{\partial}(M, \omega \text {-e-reg })_{\mathrm{ew}}^{*}$ and this yields a strong deformation retraction of $\mathcal{H}(M, \omega$-e-reg) onto $\mathcal{H}(M, \omega)$.

In this article we use a similar strategy and investigate the internal structure of the group $\mathcal{H}(M, \omega)$. The group $\mathcal{H}_{E_{M}}(M, \omega)$ acts continuously on $\mathcal{S}(M, \omega)$ by $h \cdot a=c_{h}^{\omega}+a\left(h \in \mathcal{H}_{E_{M}}(M, \omega), a \in \mathcal{S}(M, \omega)\right)$, and the end charge homomorphism $c^{\omega}: \mathcal{H}_{E_{M}}(M, \omega) \rightarrow \mathcal{S}(M, \omega)$ coincides with the orbit map at $0 \in \mathcal{S}(M, \omega)$. We extend the argument in [3] and show that the map $c^{\omega}$ admits a continuous (nonhomomorphic) section.

Suppose $M^{n}$ is a noncompact connected separable metrizable $n$-manifold and $\omega \in \mathcal{M}_{\mathrm{g}}^{\partial}(M)$.

Theorem 1.1. There exists a continuous map $s: \mathcal{S}(M, \omega) \rightarrow \mathcal{H}_{\partial}(M, \omega)_{1}$ such that $c^{\omega} s=\mathrm{id}$ and $s(0)=\mathrm{id}_{M}$.

TheOREM 1.2. Suppose that $P$ is any topological space and that $\mu$ : $P \rightarrow \mathcal{M}_{\mathrm{g}}^{\partial}\left(M, \omega\right.$-reg) and $a: P \rightarrow \mathcal{S}\left(E_{M}\right)$ are continuous maps such that $a_{p} \in \mathcal{S}\left(M, \mu_{p}\right)(p \in P)$. Then there exists a continuous map $h$ : $P \rightarrow \mathcal{H}_{\partial}(M, \omega-\text { reg })_{1}$ such that for each $p \in P$,

(1) $h_{p} \in \mathcal{H}_{\partial}\left(M, \mu_{p}\right)_{1}$,

(2) $c_{h_{p}}^{\mu_{p}}=a_{p}$

(3) if $a_{p}=0$, then $h_{p}=\operatorname{id}_{M}$.

Theorem 1.2 is a slight generalization of Theorem 1.1. The existence of a section for the map $c^{\omega}$ and the contractibility of the base space $\mathcal{S}(M, \omega)$ imply the following consequences.

Corollary 1.1.

(1) $\mathcal{H}_{E_{M}}(M, \omega) \cong \operatorname{Ker} c^{\omega} \times \mathcal{S}(M, \omega)$.

(2) $\operatorname{Ker} c^{\omega}$ is a strong deformation retract of $\mathcal{H}_{E_{M}}(M, \omega)$. 
The group $\operatorname{Ker} c^{\omega}$ contains the subgroup $\mathcal{H}^{\mathrm{c}}(M, \omega)$ consisting of $\omega$-preserving homeomorphisms with compact support. The condition $c_{h}^{\omega}=0$ means that any compact part of $h$ can be separated from the "remaining part" of $h$. From the argument in [1] it follows that for any $f \in \operatorname{Ker} c^{\omega} \cap \mathcal{H}(M)_{1}$ and any compact subset $K$ of $M$ there exists a compact connected $n$-submanifold $N$ of $M$ with $K \subset N$ and $h \in \mathcal{H}_{M-N}(M, \omega)_{1}$ with $\left.h\right|_{K}=\left.f\right|_{K}$. This implies that the subgroup $\mathcal{H}^{\mathrm{c}}(M, \omega)_{1}^{*}$ is dense in $\operatorname{Ker} c^{\omega} \cap \mathcal{H}(M)_{1}$. In a succeeding work we will show that in $n=2$ the subgroup $\mathcal{H}^{\mathrm{c}}(M, \omega)_{1}^{*}$ is homotopy dense in $\operatorname{Ker} c^{\omega} \cap \mathcal{H}(M)_{1}$. In [9] we have obtained some versions of Theorem 1.1 and [3, Theorem 4.1] for smooth manifolds and volume-preserving diffeomorphisms.

This paper is organized as follows. Section 2 contains fundamentals on end compactifications, spaces of Radon measures and groups of measurepreserving homeomorphisms. Section 3 is devoted to basics on end charge homomorphisms and related notions. This section also includes generalities on morphisms induced from proper maps. Section 4 contains the proof of Theorem 1.2 in the cube case. The general case is treated in Section 5.

\section{Spaces of Radon measures and groups of homeomorphisms.} Throughout this section $X$ is a connected, locally connected, locally compact, separable metrizable space. We use the notations $\mathcal{F}(X), \mathcal{K}(X)$ and $\mathcal{C}(X)$ for the sets of closed subsets, compact subsets, and connected components of $X ; \mathcal{B}(X)$ and $\mathcal{Q}(X)$ denote the $\sigma$-algebra of Borel subsets and the algebra of clopen subsets of $X$ respectively.

When $A$ is a subset of $X$, the symbols $\operatorname{Fr}_{X} A, \operatorname{cl}_{X} A$ and $\operatorname{Int}_{X} A$ denote the frontier, closure and interior of $A$ relative to $X$. When $M$ is a manifold, $\partial=\partial M$ and Int $M$ denote the boundary and interior of $M$ as a manifold.

2.1. Groups of homeomorphisms. For a space $X$ and a subset $A \subset X$ the symbol $\mathcal{H}_{A}(X)$ denotes the group of homeomorphisms $h$ of $X$ onto itself with $\left.h\right|_{A}=\operatorname{id}_{A}$, equipped with the compact-open topology. The group $\mathcal{H}_{A}(X)$ is a topological group (since $X$ is locally compact and locally connected).

The support of $h \in \mathcal{H}(X)$ is defined by $\operatorname{Supp} h=\operatorname{cl}_{X}\{x \in X \mid h(x) \neq x\}$. We set $\mathcal{H}_{A}^{\mathrm{c}}(X)=\left\{h \in \mathcal{H}_{A}(X) \mid \operatorname{Supp} h\right.$ compact $\}$. For any subgroup $\mathcal{G}$ of $\mathcal{H}(X)$, the symbol $\mathcal{G}_{1}$ denotes the path-component of $\operatorname{id}_{M}$ in $\mathcal{G}$. When $\mathcal{G} \subset \mathcal{H}^{\mathrm{c}}(X)$, we denote by $\mathcal{G}_{1}^{*}$ the subgroup of $\mathcal{G}_{1}$ consisting of those $h \in \mathcal{G}$ which admit an isotopy $h_{t} \in \mathcal{G}(t \in[0,1])$ such that $h_{0}=\operatorname{id}_{X}, h_{1}=h$ and there exists $K \in \mathcal{K}(X)$ with $\operatorname{Supp} h_{t} \subset K(t \in[0,1])$.

2.2. End compactifications (cf. $[1,3]$ ). Suppose $X$ is a noncompact, connected, locally connected, locally compact, separable metrizable space. An end of $X$ is a function $e$ which assigns an $e(K) \in \mathcal{C}(X-K)$ to each $K \in \mathcal{K}(X)$ so that $e\left(K_{1}\right) \supset e\left(K_{2}\right)$ if $K_{1} \subset K_{2}$. The set of ends of $X$ is denoted by $E_{X}$. 
The end compactification of $X$ is the space $\bar{X}=X \cup E_{X}$ equipped with the topology defined by the following conditions: (i) $X$ is an open subspace of $\bar{X}$, (ii) the fundamental open neighborhoods of $e \in E_{X}$ are given by

$$
N(e, K)=e(K) \cup\left\{e^{\prime} \in E_{X} \mid e^{\prime}(K)=e(K)\right\} \quad(K \in \mathcal{K}(X)) .
$$

Then $\bar{X}$ is a connected, locally connected, compact, metrizable space, $X$ is a dense open subset of $\bar{X}$, and $E_{X}$ is a compact 0-dimensional subset of $\bar{X}$.

Let $\mathcal{B}_{\mathrm{c}}(X)=\left\{C \in \mathcal{B}(X) \mid \operatorname{Fr}_{X} C\right.$ compact $\}$. For each $C \in \mathcal{B}_{\mathrm{c}}(X)$ let

$$
E_{C}=\left\{e \in E_{X} \mid e(K) \subset C \text { for some } K \in \mathcal{K}(X)\right\}, \quad \bar{C}=C \cup E_{C} \subset \bar{X} .
$$

Then $E_{C} \in \mathcal{Q}\left(E_{X}\right)$ and $\bar{C}$ is a neighborhood of $E_{C}$ in $\bar{X}$ with $\bar{C} \cap E_{X}=E_{C}$. For $C, D \in \mathcal{B}_{\mathrm{c}}(X), E_{C}=E_{D}$ iff $C \triangle D=(C-D) \cup(D-C)$ is relatively compact (i.e., has compact closure) in $X$.

For $h \in \mathcal{H}(X)$ and $e \in E_{X}$ we define $h(e) \in E_{X}$ by $h(e)(K)=$ $h\left(e\left(h^{-1}(K)\right)\right)(K \in \mathcal{K}(X))$. Each $h \in \mathcal{H}(X)$ has a unique extension $\bar{h} \in$ $\mathcal{H}(\bar{X})$ defined by $\bar{h}(e)=h(e)\left(e \in E_{X}\right)$. The map $\mathcal{H}(X) \rightarrow \mathcal{H}(\bar{X}): h \mapsto \bar{h}$ is a continuous group homomorphism. We set $\mathcal{H}_{A \cup E_{X}}(X)=\left\{h \in \mathcal{H}_{A}(X) \mid\right.$ $\left.\left.\bar{h}\right|_{E_{X}}=\operatorname{id}_{E_{X}}\right\}$. Note that $\mathcal{H}_{A \cup E_{X}}(X)_{1}=\mathcal{H}_{A}(X)_{1}$ and if $C \in \mathcal{B}_{\mathrm{c}}(X)$ and $h \in \mathcal{H}_{E_{X}}(X)$, then $h(C) \in \mathcal{B}_{\mathrm{c}}(X)$ and $E_{h(C)}=E_{C}$.

2.3. Space of Radon measures. Next we recall general facts on spaces of Radon measures (cf. [1, 3, 6]). Suppose $X$ is a connected, locally connected, locally compact, separable metrizable space. A Radon measure on $X$ is a measure $\mu$ on $(X, \mathcal{B}(X))$ such that $\mu(K)<\infty$ for any $K \in \mathcal{K}(X)$. A Radon measure $\mu$ on $X$ is said to be good if $\mu(p)=0$ for any point $p \in X$ and $\mu(U)>0$ for any nonempty open subset $U$ of $X$.

Let $\mathcal{M}(X)$ denote the space of Radon measures $\mu$ on $X$ equipped with the weak topology. This topology is the weakest topology such that the function

$$
\Phi_{f}: \mathcal{M}(X) \rightarrow \mathbb{R}, \quad \Phi_{f}(\mu)=\int_{X} f d \mu,
$$

is continuous for any continuous function $f: X \rightarrow \mathbb{R}$ with compact support. Let $\mathcal{M}_{\mathrm{g}}(X)$ denote the subspace of good Radon measures $\mu$ on $X$ and for $A \in \mathcal{B}(X)$ we set $\mathcal{M}^{A}(X)=\{\mu \in \mathcal{M}(X) \mid \mu(A)=0\}$ and $\mathcal{M}_{\mathrm{g}}^{A}(X)=$ $\mathcal{M}_{\mathrm{g}}(X) \cap \mathcal{M}^{A}(X)$.

For $\mu \in \mathcal{M}(X)$ and $A \in \mathcal{B}(X)$ the restriction $\left.\mu\right|_{A} \in \mathcal{M}(A)$ is defined by $\left(\left.\mu\right|_{A}\right)(B)=\mu(B)(B \in \mathcal{B}(A))$.

Lemma 2.1 ([3, Lemma 2.2]). Let $A \in \mathcal{F}(X)$ and $K \in \mathcal{K}(X)$.

(i) The restriction map $\mathcal{M}^{\mathrm{Fr} A}(X) \rightarrow \mathcal{M}(A):\left.\mu \mapsto \mu\right|_{A}$ is continuous.

(ii) The evaluation map $\mathcal{M}^{\operatorname{Fr} K}(X) \rightarrow \mathbb{R}: \mu \mapsto \mu(K)$ is continuous. 
Let $\omega \in \mathcal{M}_{\mathrm{g}}(X)$. We say that an end $e \in E_{X}$ is $\omega$-finite if $\omega(e(K))<\infty$ for some $K \in \mathcal{K}(X)$. Let $E_{X}^{\omega}=\left\{e \in E_{X} \mid e\right.$ is $\omega$-finite $\}$. This is an open subset of $E_{X}$ and for $C \in \mathcal{B}_{\mathrm{c}}(X)$ we have $E_{C} \subset E_{X}^{\omega}$ iff $\omega(C)<\infty$.

DEFinition 2.1.

(1) $\mu \in \mathcal{M}_{\mathrm{g}}(X)$ is said to be

(i) $\omega$-regular if $\mu$ has the same null sets as $\omega$ (i.e., $\mu(B)=0$ iff $\omega(B)=0$ for any $B \in \mathcal{B}(X)$ ),

(ii) $\omega$-end-regular if $\mu$ is $\omega$-regular and $E_{X}^{\mu}=E_{X}^{\omega}$.

(2) $\mathcal{M}_{g}^{A}\left(X, \omega\left(\right.\right.$-e)-reg) $=\left\{\mu \in \mathcal{M}_{g}^{A}(X) \mid \mu\right.$ is $\omega$ (-end)-regular $\}$ (with the weak topology).

The group $\mathcal{H}(X)$ acts continuously on $\mathcal{M}(X)$ by $h \cdot \mu=h_{*} \mu$, where $h_{*} \mu$ is defined by $\left(h_{*} \mu\right)(B)=\mu\left(h^{-1}(B)\right)(B \in \mathcal{B}(X))$.

DeFinition 2.2.

(1) $h \in \mathcal{H}(X)$ is said to be

(i) $\omega$-preserving if $h_{*} \omega=\omega$ (i.e., $\omega(h(B))=\omega(B)$ for any $\left.B \in \mathcal{B}(X)\right)$,

(ii) $\omega$-regular if $h$ preserves $\omega$-null sets (i.e., $\omega(h(B))=0$ iff $\omega(B)=0$ for any $B \in \mathcal{B}(X))$,

(iii) $\omega$-end-regular if $h$ is $\omega$-regular and $\bar{h}\left(E_{X}^{\omega}\right)=E_{X}^{\omega}$.

(2) $\mathcal{H}(X, \omega)=\{h \in \mathcal{H}(X) \mid h$ is $\omega$-preserving $\}$ and $\mathcal{H}(X, \omega(-\mathrm{e})$-reg $)=$ $\{h \in \mathcal{H}(X) \mid h$ is $\omega$ (-end)-regular $\}$.

Suppose $M$ is a compact connected $n$-manifold. The von NeumannOxtoby-Ulam theorem [7] asserts that if $\mu, \nu \in \mathcal{M}_{\mathrm{g}}^{\partial}(M)$ and $\mu(M)=\nu(M)$, then there exists $h \in \mathcal{H}_{\partial}(M)_{1}$ such that $h_{*} \mu=\nu$. A. Fathi [6] obtained a parameter version of this theorem.

THEOREM 2.1. Suppose that $M$ is a compact connected $n$-manifold and $\omega \in \mathcal{M}_{\mathrm{g}}^{\partial}(M)$. Suppose that $\mu, \nu, P \rightarrow \mathcal{M}_{\mathrm{g}}^{\partial}(M, \omega-\mathrm{reg})$ are continuous maps with $\mu_{p}(M)=\nu_{p}(M)(p \in P)$. Then there exists a continuous map $h$ : $P \rightarrow \mathcal{H}_{\partial}(M, \omega-\mathrm{reg})_{1}$ such that for each $p \in P$,

(i) $\left(h_{p}\right)_{*} \mu_{p}=\nu_{p}$,

(ii) if $\mu_{p}=\nu_{p}$ then $h_{p}=\operatorname{id}_{M}$.

In [3] R. Berlanga obtained a similar theorem for a noncompact connected $n$-manifold $M$. We use the following consequence of [3, Proposition 5.1(2)].

LemMa 2.2. Suppose that $M$ is a noncompact connected $n$-manifold and $\omega \in \mathcal{M}_{\mathrm{g}}^{\partial}(M)$. Then $\mathcal{H}_{\partial}(M, \omega) \cap \mathcal{H}_{\partial}(M, \omega-\mathrm{reg})_{1}=\mathcal{H}_{\partial}(M, \omega)_{1}$. 


\section{End charge homomorphism}

3.1. End charge homomorphism. We recall the basic properties of the end charge homomorphism defined in [1, Section 14]. Suppose $X$ is a connected, locally connected, locally compact, separable metrizable space and $\omega \in \mathcal{M}(X)$.

An end charge of $X$ is a finitely additive signed measure $c$ on $\mathcal{Q}\left(E_{X}\right)$, that is, a function $c: \mathcal{Q}\left(E_{X}\right) \rightarrow \mathbb{R}$ which satisfies the following condition:

$$
c(F \cup G)=c(F)+c(G) \quad \text { for } F, G \in \mathcal{Q}\left(E_{X}\right) \text { with } F \cap G=\emptyset .
$$

Let $\mathcal{S}\left(E_{X}\right)$ denote the space of end charges $c$ of $X$ with the weak topology (or the product topology). This topology is the weakest topology such that the function

$$
\Psi_{F}: \mathcal{S}\left(E_{X}\right) \rightarrow \mathbb{R}, \quad \Psi_{F}(c)=c(F),
$$

is continuous for any $F \in \mathcal{Q}\left(E_{X}\right)$. For a subset $U \subset E_{X}$ let

$$
\mathcal{S}_{0}\left(E_{X}, U\right)=\left\{\begin{array}{l|l}
c \in \mathcal{S}\left(E_{X}\right) \mid \begin{array}{l}
\text { (i) } c(F)=0 \text { for } F \in \mathcal{Q}\left(E_{X}\right) \text { with } F \subset U, \\
\text { (ii) } c\left(E_{X}\right)=0
\end{array}
\end{array}\right\}
$$

(with the weak topology). Then $\mathcal{S}\left(E_{X}\right)$ is a topological linear space and $\mathcal{S}_{0}\left(E_{X}, U\right)$ is a linear subspace. For $\omega \in \mathcal{M}(X)$ we set $\mathcal{S}(X, \omega)=\mathcal{S}_{0}\left(E_{X}, E_{X}^{\omega}\right)$.

For $h \in \mathcal{H}_{E_{X}}(X, \omega)$ the end charge $c_{h}^{\omega} \in \mathcal{S}(X, \omega)$ is defined as follows: For any $F \in \mathcal{Q}\left(E_{X}\right)$ there exists $C \in \mathcal{B}_{\mathrm{c}}(X)$ with $E_{C}=F$. Since $\left.\bar{h}\right|_{E_{X}}=\mathrm{id}$, it follows that $E_{C}=E_{h(C)}$ and $C \triangle h(C)$ is relatively compact in $X$. Thus $\omega(C-h(C)), \omega(h(C)-C)<\infty$ and we can define

$$
c_{h}^{\omega}(F)=\omega(C-h(C))-\omega(h(C)-C) \in \mathbb{R} .
$$

This quantity is independent of the choice of $C$.

Proposition 3.1. The map $c^{\omega}: \mathcal{H}_{E_{X}}(X, \omega) \rightarrow \mathcal{S}(X, \omega)$ is a continuous group homomorphism ([1, Section 14.9, Lemma 14.21(iv)]).

3.2. Related notions. In the proof of Theorem 1.2 it is necessary to measure volumes transferred into various regions by homeomorphisms (which are not measure-preserving). For this purpose we introduce some notation.

For $A, B \in \mathcal{B}(X)$ we write $A \sim_{\mathrm{c}} B$ if $A \triangle B$ is relatively compact in $X$. This is an equivalence relation and for $A, B \in \mathcal{B}_{\mathrm{c}}(X)$ we have

(i) $A \sim_{\mathrm{c}} B$ iff $E_{A}=E_{B}$,

(ii) $A \sim_{\mathrm{c}} h(A)$ for any $h \in \mathcal{H}_{E_{X}}(X)$.

Similarly, for $\mu \in \mathcal{M}(X)$ and $A, B \in \mathcal{B}(X)$ we write $A \sim_{\mu} B$ if $\mu(A \triangle B)<\infty$. This is also an equivalence relation and $A \sim_{\mathrm{c}} B$ implies $A \sim_{\mu} B$. If $A \sim_{\mu} B$, then we can consider the following quantity:

$$
J^{\mu}(A, B)=\mu(A-B)-\mu(B-A) \in \mathbb{R} .
$$


It measures the difference of $\mu$-volumes of $A$ and $B$ when $A$ and $B$ differ only in a finite volume part. If $C \in \mathcal{B}_{\mathrm{c}}(X)$ and $h \in \mathcal{H}_{E_{X}}(X)$, then $J^{\mu}\left(h^{-1}(C), C\right)$ is just the total $\mu$-mass transferred into $C$ by $h$. If $h \in \mathcal{H}_{E_{X}}(X, \mu)$, then $J^{\mu}\left(h^{-1}(C), C\right)=J^{\mu}(C, h(C))=c_{h}^{\mu}\left(E_{C}\right)$.

This quantity has the following formal properties:

Lemma 3.1. Suppose $\mu \in \mathcal{M}(X)$ and $A, B, C, D \in \mathcal{B}(X)$.

(1) If $A \sim_{\mu} B$ and $\mu(A)<\infty$, then $\mu(B)<\infty$ and

$$
J^{\mu}(A, B)=\mu(A)-\mu(B) .
$$

(2) If $A \sim_{\mu} B \sim_{\mu} C$, then $J^{\mu}(A, B)+J^{\mu}(B, C)=J^{\mu}(A, C)$.

(3) If $A \sim_{\mu} C, B \sim_{\mu} D$, then

(i) $A \cup B \sim_{\mu} C \cup D$ since $(A \cup B) \triangle(C \cup D) \subset(A \triangle C) \cup(B \triangle D)$,

(ii) if $A \cap B=C \cap D=\emptyset$, then

$$
J^{\mu}(A \cup B, C \cup D)=J^{\mu}(A, C)+J^{\mu}(B, D) .
$$

(4) If $h \in \mathcal{H}(X)$ and $A \sim_{h_{*} \mu} B$, then $h^{-1}(A) \sim_{\mu} h^{-1}(B)$ and

$$
J^{h_{*} \mu}(A, B)=J^{\mu}\left(h^{-1}(A), h^{-1}(B)\right) .
$$

Lemma 3.2. Suppose $\omega \in \mathcal{M}(X)$ and $A, B \in \mathcal{B}_{\mathrm{c}}(X)$ with $A \sim_{\mathrm{c}} B$ and $\omega(\operatorname{Fr} A)=\omega(\operatorname{Fr} B)=0$. Then the function

$$
\Phi: \mathcal{M}(X, \omega \text {-reg }) \times \mathcal{H}_{E_{X}}(X, \omega-\text { reg })^{2} \rightarrow \mathbb{R}, \quad \Phi(\mu, f, g)=J^{\mu}(f(A), g(B)),
$$

is continuous.

Proof. Since $J^{\mu}(f(A), g(B))=J^{\mu}(f(A), A)+J^{\mu}(A, B)+J^{\mu}(B, g(B))$ and $\mu(A-f(A))=\left(f_{*}^{-1} \mu\right)\left(f^{-1}(A)-A\right)$, it suffices to verify the continuity of the following function:

$$
\mathcal{M}(X, \omega \text {-reg }) \times \mathcal{H}_{E_{X}}(X, \omega \text {-reg }) \rightarrow \mathbb{R}:(\mu, f) \mapsto \mu(f(A)-A) .
$$

Fix $(\mu, f)$ and $\varepsilon>0$. Since $f(\operatorname{Fr} A)$ is a compact $\mu$-null set, it has a compact neighborhood $K$ such that $\mu(K)<\varepsilon$. There exists a neighborhood $\mathcal{U}$ of $f$ in $\mathcal{H}_{E_{X}}(X, \omega$-reg) such that $f(A) \triangle g(A) \subset K(g \in \mathcal{U})$.

The function $\nu(f(A)-A)$ is continuous in $\nu$. In fact, $\operatorname{Fr}(f(A)-A) \subset$ $\operatorname{Fr} A \cup \operatorname{Fr} f(A)$ and the latter is a $\nu$-null set since $\nu$ is $\omega$-regular. Thus, we have $\nu(\operatorname{Fr}(f(A)-A))=0$ and the claim follows from Lemma 2.1(ii). Also note that the function $\mathcal{M}(X) \rightarrow \mathbb{R}: \nu \mapsto \nu(K)$ is upper semicontinuous ([3, Lemma 2.1]). Therefore, there exists a neighborhood $\mathcal{V}$ of $\mu$ in $\mathcal{M}(X, \omega$-reg $)$ such that

$$
|\nu(f(A)-A)-\mu(f(A)-A)|<\varepsilon \quad \text { and } \quad \nu(K)<\varepsilon \quad(\nu \in \mathcal{V}) .
$$

Take any $(\nu, g) \in \mathcal{V} \times \mathcal{U}$. Since $(f(A)-A) \triangle(g(A)-A) \subset f(A) \triangle g(A) \subset K$, 
we have

$$
|\nu(g(A)-A)-\nu(f(A)-A)| \leq \nu(K)<\varepsilon .
$$

(In general, $|\nu(A)-\nu(B)| \leq \nu(A \triangle B)$.) It follows that

$$
|\nu(g(A)-A)-\mu(f(A)-A)|<2 \varepsilon
$$

Following [3] we say that continuous maps $\mu, \nu: P \rightarrow \mathcal{M}(X)$ are compactly related and write $\mu \sim_{\mathrm{c}} \nu$ if each $p \in P$ admits a neighborhood $U$ in $P$ and $K_{p} \in \mathcal{K}(X)$ such that $\mu_{q}=\nu_{q}$ on $M-K_{p}(q \in U)$. (If $P$ is a singleton, this is just a condition on $\mu, \nu \in \mathcal{M}(X)$.) This is an equivalence relation and if $\mu \sim_{\mathrm{c}} \nu$, then for any $C \in \mathcal{B}(X)$ we can define a function $(\mu-\nu)(C): P \rightarrow \mathbb{R}$ by

$$
(\mu-\nu)(C)_{p}=\mu_{p}\left(C \cap K_{p}\right)-\nu_{p}\left(C \cap K_{p}\right)
$$

This definition is independent of the choice of $K_{p}$. If $\omega \in \mathcal{M}(X), \mu, \nu: P \rightarrow$ $\mathcal{M}(X, \omega$-reg $), \mu \sim_{\mathrm{c}} \nu$ and $C \in \mathcal{B}(X)$ with $\omega(\operatorname{Fr} C)=0$, then the function $(\mu-\nu)(C): P \rightarrow \mathbb{R}$ is continuous.

Suppose a continuous map $h: P \rightarrow \mathcal{H}^{\mathrm{c}}(X)$ has locally common compact support (i.e., for each $p \in P$ there exists a neighborhood $U$ of $p$ in $P$ and $K \in \mathcal{K}(X)$ such that $\left.\operatorname{Supp} h_{q} \subset K(q \in U)\right)$. Then $\mu \sim_{\mathrm{c}} h_{*} \mu$ for any continuous map $\mu: P \rightarrow \mathcal{M}(X)$.

If $\mu \in \mathcal{M}(X), A \in \mathcal{B}(X)$ and $f, g \in \mathcal{H}^{\mathrm{c}}(X)$, then

$$
\left(f_{*} \mu-g_{*} \mu\right)(A)=J^{\mu}\left(f^{-1}(A), g^{-1}(A)\right) .
$$

In the proof of Theorem 1.2 we frequently use the quantity on the right hand side. The above means that this quantity can be translated to a quantity prescribed in terms of measures and that the calculations on this quantity in the proof of Theorem 1.2 and the statements in Lemma 3.1 reduce to calculations on measures. However, $J^{\mu}\left(f^{-1}(A), g^{-1}(A)\right)$ has the advantage that it is defined for $A \in \mathcal{B}_{\mathrm{c}}(X)$ and $f, g \in \mathcal{H}_{E_{X}}(X)$. For example, we can take $f$ and $g$ to be the limits of sequences $f_{k}, g_{k} \in \mathcal{H}^{\mathrm{c}}(X)$. This is convenient in our situation.

3.3. Morphisms induced from proper maps. Suppose $X$ and $Y$ are connected, locally connected, locally compact, separable metrizable spaces and $f: X \rightarrow Y$ is a proper continuous map (that is, $f^{-1}(K)$ is compact for any $K \in \mathcal{K}(Y))$. The map $f$ induces various continuous morphisms.

(1) $f_{*}: \mathcal{M}(X) \rightarrow \mathcal{M}(Y)$ : For $\mu \in \mathcal{M}(X)$ the induced measure $f_{*} \mu \in \mathcal{M}(Y)$ is defined by $\left(f_{*} \mu\right)(B)=\mu\left(f^{-1}(B)\right)(B \in \mathcal{B}(Y))$. The map $f_{*}$ is continuous. If $A, B \in \mathcal{B}(Y)$ and $A \sim_{f_{*} \mu} B$, then $J^{f_{*} \mu}(A, B)=$ $J^{\mu}\left(f^{-1}(A), f^{-1}(B)\right)$ (cf. Lemma 3.1(4)). 
(2) $\bar{f}: \bar{X} \rightarrow \bar{Y}$ : This is the unique continuous extension of $f$. For each $e \in E_{X}$ the end $f(e) \in E_{Y}$ is defined by assigning to each $K \in \mathcal{K}(Y)$ the unique component $f(e)(K) \in \mathcal{C}(Y-K)$ which contains $f\left(e\left(f^{-1}(K)\right)\right)$. The map $\bar{f}$ is defined by $\bar{f}(e)=f(e)\left(e \in E_{X}\right)$. For any $C \in \mathcal{B}_{\mathrm{c}}(Y)$ we have $f^{-1}(C) \in \mathcal{B}_{\mathrm{c}}(X)$ and $E_{f^{-1}(C)}=\bar{f}^{-1}\left(E_{C}\right)$.

(3) $\bar{f}_{*}: \mathcal{S}\left(E_{X}\right) \rightarrow \mathcal{S}\left(E_{Y}\right)$ : This is a continuous linear map induced by the map $\bar{f}: E_{X} \rightarrow E_{Y}$. For each $c \in \mathcal{S}\left(E_{X}\right)$ the end charge $\bar{f}_{*} c \in$ $\mathcal{S}\left(E_{Y}\right)$ is defined by $\left(\bar{f}_{*} c\right)(F)=c\left(\bar{f}^{-1}(F)\right)\left(F \in \mathcal{Q}\left(E_{Y}\right)\right)$. It induces the restriction $\bar{f}_{*}: \mathcal{S}_{0}\left(E_{X}, U\right) \rightarrow \mathcal{S}_{0}\left(E_{Y}, V\right)$ for any $V \subset E_{Y}$ and $U \subset E_{X}$ with $\bar{f}^{-1}(V) \subset U$. Let $\omega \in \mathcal{M}(X)$. Since $\bar{f}^{-1}\left(E_{Y}^{f_{*} \omega}\right) \subset E_{X}^{\omega}$, we obtain the restriction $\bar{f}_{*}: \mathcal{S}(X, \omega) \rightarrow \mathcal{S}\left(Y, f_{*} \omega\right)$. If $\bar{f}: E_{X} \rightarrow E_{Y}$ is injective, then $\bar{f}^{-1}\left(E_{Y}^{f_{*} \omega}\right)=E_{X}^{\omega}$. Therefore, if $\bar{f}: E_{X} \rightarrow E_{Y}$ is a homeomorphism, then $\bar{f}_{*}: \mathcal{S}(X, \omega) \rightarrow \mathcal{S}\left(Y, f_{*} \omega\right)$ is also a homeomorphism.

Below we assume that the map $f: X \rightarrow Y$ satisfies the following additional conditions:

$(*)_{1} C \in \mathcal{F}(X), \operatorname{Int}_{X} C=\emptyset$ and $D \in \mathcal{F}(Y)$,

$(*)_{2} f(C)=D$ and $f$ maps $X-C$ homeomorphically onto $Y-D$.

(4) $f^{*}: \mathcal{M}^{D}(Y) \rightarrow \mathcal{M}^{C}(X)$ : For each $\nu \in \mathcal{M}^{D}(Y)$ the measure $f^{*} \nu \in \mathcal{M}^{C}(X)$ is defined by $\left(f^{*} \nu\right)(B)=\nu(f(B-C))(B \in \mathcal{B}(X))$. The map $f^{*}$ is a homeomorphism, whose inverse is the map $f_{*}: \mathcal{M}^{C}(X) \rightarrow$ $\mathcal{M}^{D}(Y)$. For any $\omega \in \mathcal{M}_{\mathrm{g}}^{D}(Y)$ these maps induce the reciprocal homeomorphisms

$$
\begin{aligned}
& f_{*}: \mathcal{M}_{\mathrm{g}}^{C}\left(X, f^{*} \omega-\mathrm{reg}\right) \rightarrow \mathcal{M}_{\mathrm{g}}^{D}(Y, \omega-\mathrm{reg}), \\
& f^{*}: \mathcal{M}_{\mathrm{g}}^{D}(Y, \omega-\text { reg }) \rightarrow \mathcal{M}_{\mathrm{g}}^{C}\left(X, f^{*} \omega \text {-reg }\right) .
\end{aligned}
$$

(5) $f_{*}: \mathcal{H}_{C}(X) \rightarrow \mathcal{H}_{D}(Y)$ : For each $h \in \mathcal{H}_{C}(X)$ there exists a unique $\underline{h} \in \mathcal{H}_{D}(Y)$ such that $\underline{h} f=f h$. The map $f_{*}$ is defined by $f_{*} h=\underline{h}$. This map is a continuous injection and induces the restrictions

$$
\begin{aligned}
& f_{*}: \mathcal{H}_{C \cup E_{X}}(X) \rightarrow \mathcal{H}_{D \cup E_{Y}}(Y), \quad f_{*}: \mathcal{H}_{C}\left(X, f^{*} \omega \text {-reg }\right) \rightarrow \mathcal{H}_{D}(Y, \omega \text {-reg }), \\
& f_{*}: \mathcal{H}_{C}\left(X, f^{*} \omega\right) \rightarrow \mathcal{H}_{D}(Y, \omega), \quad \text { for any } \omega \in \mathcal{M}^{D}(Y) .
\end{aligned}
$$

LEMmA 3.3. Under the condition $(*)$, for any $\omega \in \mathcal{M}^{D}(Y)$ we have the following commutative diagram:

$$
\begin{array}{ccc}
\mathcal{H}_{C \cup E_{X}}\left(X, f^{*} \omega\right) & \stackrel{c^{f^{*} \omega}}{\longrightarrow} & \mathcal{S}\left(X, f^{*} \omega\right) \\
f_{*} \downarrow & \bar{f}_{*} \\
\mathcal{H}_{D \cup E_{Y}}(Y, \omega) & \stackrel{c^{\omega}}{\longrightarrow} & \mathcal{S}(Y, \omega)
\end{array}
$$


4. Proof of Theorem 1.2 in the cube case. In this section we prove Theorem 1.2 in the cube case. Following [3, Section 4] we use the following notations: $I=[0,1], I^{n}$ is the $n$-fold product of $I, I_{1}=[1 / 3,2 / 3] \times$ $\{(1 / 2, \ldots, 1 / 2,1)\} \subset I^{n}, m$ is the Lebesgue measure on $\mathbb{R}^{n}, d$ is the standard Euclidean distance in $\mathbb{R}^{n}(d(\mathbf{x}, \mathbf{y})=\|\mathbf{x}-\mathbf{y}\|), E$ is a 0 -dimensional compact subset of $\partial I^{n}\left(E \subset I_{1}\right.$ for $\left.n \geq 2\right), M_{0}=I^{n}-E$ and $m_{0}=\left.m\right|_{M_{0}}$. The pair $\left(\bar{M}_{0}, \bar{E}_{M_{0}}\right)$ is canonically identified with $\left(I^{n}, E\right)$. An $n$-cubic balloon in $I^{n}$ is a cube $A$ of the form $[0, \alpha]^{n}+\mathbf{v}$ for some $\alpha>0$ and $\mathbf{v} \in \mathbb{R}^{n}$ such that $A \subset I^{n}$ and $A \cap \partial I^{n}=\left([0, \alpha]^{n-1} \times\{\alpha\}\right)+\mathbf{v}$. Let $\mathcal{D}\left(M_{0}\right)$ denote the set of PL $n$-disks $K$ in $M_{0}$ such that $\operatorname{cl}_{I^{n}}\left(M_{0}-K\right)$ is a finite disjoint union of $n$-cubic balloons $A$ in $I^{n}$ with $A \cap E \neq \emptyset$. For convenience, we add the empty set $\emptyset$ as a member of $\mathcal{D}\left(M_{0}\right)$.

Theorem $1.2^{\prime}$. Suppose $\mu: P \rightarrow \mathcal{M}_{\mathrm{g}}^{\partial}\left(M_{0}, m_{0}\right.$-reg) and $a: P \rightarrow \mathcal{S}\left(E_{M_{0}}\right)$ are continuous maps such that $a_{p} \in \mathcal{S}\left(M_{0}, \mu_{p}\right)(p \in P)$. Then there exists a continuous map $h: P \rightarrow \mathcal{H}_{\partial}\left(M_{0}, m_{0} \text {-reg }\right)_{1}$ such that for each $p \in P$,

(1) $h_{p} \in \mathcal{H}_{\partial}\left(M_{0}, \mu_{p}\right)_{1}$,

(2) $c_{h_{p}}^{\mu_{p}}=a_{p}$

(3) if $a_{p}=0$, then $h_{p}=\mathrm{id}_{M_{0}}$.

Theorem $1.2^{\prime}$ is proved in a series of lemmas. For the sake of notational simplicity, we write $f_{*} \mu=g_{*} \mu$ and $J^{\mu}(f(A), g(A))=a\left(E_{A}\right)$ instead of $f_{p *} \mu_{p}=g_{p *} \mu_{p}(p \in P)$ and $J^{\mu_{p}}\left(f_{p}(A), g_{p}(A)\right)=a_{p}\left(E_{A}\right)(p \in P)$.

Below we assume that $\mu: P \rightarrow \mathcal{M}_{\mathrm{g}}^{\partial}\left(M_{0}, m_{0}\right.$-reg $)$ and $a: P \rightarrow \mathcal{S}\left(E_{M_{0}}\right)$ are continuous maps such that $a_{p} \in \mathcal{S}\left(M_{0}, \mu_{p}\right)(p \in P)$. We consider the case $n \geq 2$. (The modification for $n=1$ is obvious.)

Lemma 4.1. Suppose that $K, L \in \mathcal{D}\left(M_{0}\right), K \subset \operatorname{Int}_{M_{0}} L$ and $f, g: P \rightarrow$ $\mathcal{H}_{\partial}\left(M_{0}, m_{0} \text {-reg }\right)_{1}$ are continuous maps such that

(i) $f_{*} \mu=g_{*} \mu$ on $K$,

(ii) $J^{\mu}\left(f^{-1}(A), g^{-1}(A)\right)=a\left(E_{A}\right)\left(A \in \mathcal{C}\left(\operatorname{cl}_{M_{0}}\left(M_{0}-K\right)\right)\right)$.

Then there exists a continuous map $h: P \rightarrow \mathcal{H}_{\partial \cup K}^{\mathrm{c}}\left(M_{0}, m_{0}\right.$-reg) ${ }_{1}^{*}$ such that

(1) $(h f)_{*} \mu=g_{*} \mu$ on $L$,

(2) $J^{\mu}\left((h f)^{-1}(B), g^{-1}(B)\right)=a\left(E_{B}\right)\left(B \in \mathcal{C}\left(\operatorname{cl}_{M_{0}}\left(M_{0}-L\right)\right)\right)$,

(3) $\left\{h_{p}^{-1}\right\}_{p \in P}$ is equicontinuous on $\mathrm{cl}_{M_{0}}\left(M_{0}-L\right)$ with respect to $\left.d\right|_{M_{0}}$,

(4) if $p \in P, a_{p}=0$ and $f_{p}=g_{p}=\operatorname{id}_{M_{0}}$, then $h_{p}=\operatorname{id}_{M_{0}}$.

Proof. For each $A \in \mathcal{C}\left(\mathrm{cl}_{M_{0}}(M-K)\right)$ we construct a continuous map

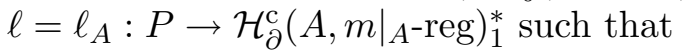


$(1)^{\prime} \quad \ell_{*}\left(\left.\left(f_{*} \mu\right)\right|_{A}\right)=g_{*} \mu$ on $A \cap L$,

$(2)^{\prime} \quad J^{\mu}\left(f^{-1} \ell^{-1}(B), g^{-1}(B)\right)=a\left(E_{B}\right)\left(B \in \mathcal{C}\left(\operatorname{cl}_{M_{0}}(A-L)\right)\right)$,

$(3)^{\prime}\left\{\ell_{p}^{-1}\right\}_{p \in P}$ is equicontinuous on $\operatorname{cl}_{M_{0}}(A-L)$ with respect to $\left.d\right|_{A}$,

$(4)^{\prime}$ if $p \in P, a_{p}=0$ and $f_{p}=g_{p}=\operatorname{id}_{M_{0}}$, then $\ell_{p}=\mathrm{id}_{A}$.

Then $h$ is defined by $\left.h\right|_{K}=\operatorname{id}_{K}$ and $\left.h\right|_{A}=\ell_{A}\left(A \in \mathcal{C}\left(\operatorname{cl}_{M_{0}}\left(M_{0}-K\right)\right)\right)$.

The map $\ell=\ell_{A}$ is constructed as follows. Let $\mathcal{C}\left(\mathrm{cl}_{M_{0}}(A-L)\right)=$ $\left\{B_{1}, \ldots, B_{m}\right\}$. This is a disjoint family of $n$-cubic balloons with ends and we have $A=(A \cap L) \cup \bigcup_{k=1}^{m} B_{k}$ and $E_{A}=\bigcup_{k=1}^{m} E_{B_{k}}$. Set $N_{k}=(A \cap L) \cup \bigcup_{i=k}^{m} B_{i}$ $(k=1, \ldots, m)$ and $N_{m+1}=A \cap L$.

We inductively construct continuous maps $\ell^{k}: P \rightarrow \mathcal{H}_{\partial}^{\mathrm{c}}\left(A,\left.m\right|_{\left.A^{-} \text {reg }\right)_{1}^{*}}\right.$ $(k=1, \ldots, m)$ such that

$\left(2_{k}\right) J^{\mu}\left(f^{-1}\left(\ell^{k}\right)^{-1}\left(B_{j}\right), g^{-1}\left(B_{j}\right)\right)=a\left(E_{B_{j}}\right)(j=1, \ldots, k)$,

$\left(3_{k}\right)\left\{\left(\ell_{p}^{k}\right)^{-1}\right\}_{p}$ is equicontinuous with respect to $\left.d\right|_{A}$,

$\left(4_{k}\right)$ if $p \in P, a_{p}=0$ and $f_{p}=g_{p}=\operatorname{id}_{M_{0}}$, then $\ell_{p}^{k}=\mathrm{id}_{A}$.

Suppose $\ell^{k-1}$ has been constructed. (For $k=1$ we put $\ell^{0} \equiv \operatorname{id}_{M_{0}}$.) Consider the PL $n$-disk $\bar{N}_{k}=\bar{B}_{k} \cup \bar{N}_{k+1}$ (recall that $\bar{N}_{k}=N_{k} \cup E_{N_{k}}=$ $\left.\operatorname{cl}_{I^{n}} N_{k}\right)$. Since $\bar{B}_{k} \cap \bar{N}_{k+1}$ is a PL $(n-1)$-disk, we can find a one-parameter family of PL-maps $\varphi_{t}: \bar{N}_{k} \rightarrow \bar{N}_{k}(t \in[-1,1])$ such that

(a) $\varphi_{0}=$ id, $\varphi_{1}\left(\bar{B}_{k}\right)=\bar{N}_{k}, \varphi_{-1}\left(\bar{N}_{k+1}\right)=\bar{N}_{k}$ and $\varphi_{t}=$ id on $\partial \bar{N}_{k}$ $(t \in[-1,1])$

(b) $\left.\varphi_{t}\right|_{N_{k}}(t \in(-1,1))$ is an isotopy on $N_{k}, \varphi_{s}\left(B_{k}\right) \subsetneq \varphi_{t}\left(B_{k}\right)(-1 \leq s$ $<t \leq 1)$ and $\left.\varphi_{t}\right|_{N_{k}}(t \in(-1,1))$ has locally common compact support.

The map $\varphi_{t}$ is obtained by enlarging $\bar{B}_{k}$ for $t \geq 0$ (engulfing $\bar{N}_{k}$ at $t=1$ ) and shrinking $\bar{B}_{k}$ for $t \leq 0$ (collapsing at $t=-1$ ). The family $\varphi_{t}(t \in[-1,1]$ ) is equicontinuous with respect to $d$, since it is a compact family. Thus $\left.\varphi_{t}\right|_{N_{k}}(t \in(-1,1))$ is also equicontinuous with respect to $d$. The maps $\varphi_{t}$ $(t \in(-1,1))$ are $m$-regular since any PL-homeomorphism between subpolyhedra in $\mathbb{R}^{n}$ is $m$-regular.

The map $\ell^{k}$ is defined as $\ell^{k}=\psi \ell^{k-1}$, where

$$
\psi: P \rightarrow \mathcal{H}_{\partial \cup B_{1} \cup \cdots \cup B_{k-1}}^{\mathrm{c}}\left(A,\left.m\right|_{A}-\mathrm{reg}\right)_{1}^{*}
$$

is defined by

$$
\psi_{p}=\varphi_{t(p)}^{-1} \quad \text { on } N_{k}, \quad \psi_{p}=\mathrm{id} \quad \text { on } B_{1} \cup \cdots \cup B_{k-1} .
$$

The parameter function $t=t(p): P \rightarrow(-1,1)$ is determined by the condition $\left(2_{k}\right)(j=k)$. We set

$$
\sigma_{p}^{k-1} \equiv\left(\ell_{p}^{k-1}\right)_{*}\left(\left.\left(f_{p_{*}} \mu_{p}\right)\right|_{A}\right) \in \mathcal{M}_{\mathrm{g}}^{\partial}\left(A,\left.m\right|_{A^{-}} \mathrm{reg}\right) .
$$


Then the identity for $j=k$ in the condition $\left(2_{k}\right)$ is equivalent to:

$$
\begin{aligned}
& a_{p}\left(E_{B_{k}}\right)-J^{\mu_{p}}\left(f_{p}^{-1}\left(\ell_{p}^{k-1}\right)^{-1}\left(B_{k}\right), g_{p}^{-1}\left(B_{k}\right)\right) \\
& =J^{\mu_{p}}\left(f_{p}^{-1}\left(\ell_{p}^{k}\right)^{-1}\left(B_{k}\right), f_{p}^{-1}\left(\ell_{p}^{k-1}\right)^{-1}\left(B_{k}\right)\right) \\
& =J^{\mu_{p}}\left(f_{p}^{-1}\left(\ell_{p}^{k-1}\right)^{-1} \varphi_{t}\left(B_{k}\right), f_{p}^{-1}\left(\ell_{p}^{k-1}\right)^{-1}\left(B_{k}\right)\right) \\
& =J^{\sigma_{p}^{k-1}}\left(\varphi_{t}\left(B_{k}\right), B_{k}\right) \\
& = \begin{cases}\sigma_{p}^{k-1}\left(\varphi_{t}\left(B_{k}\right)-B_{k}\right) \in\left[0, \sigma_{p}^{k-1}\left(N_{k+1}\right)\right) & (t \in[0,1)), \\
-\sigma_{p}^{k-1}\left(B_{k}-\varphi_{t}\left(B_{k}\right)\right) \in\left(-\sigma_{p}^{k-1}\left(B_{k}\right), 0\right] & (t \in(-1,0]) .\end{cases}
\end{aligned}
$$

(Note that $a_{p}\left(E_{B_{k}}\right)=0$ does not imply $t(p)=0$.) This equation in $t$ is uniquely solved, once we check the next inequality:

$$
a_{p}\left(E_{B_{k}}\right)-J^{\mu_{p}}\left(f_{p}^{-1}\left(\ell_{p}^{k-1}\right)^{-1}\left(B_{k}\right), g_{p}^{-1}\left(B_{k}\right)\right) \in\left(-\sigma_{p}^{k-1}\left(B_{k}\right), \sigma_{p}^{k-1}\left(N_{k+1}\right)\right) .
$$

This is verified by the following observations:

If $\sigma_{p}^{k-1}\left(B_{k}\right)=\mu_{p}\left(f_{p}^{-1}\left(\ell_{p}^{k-1}\right)^{-1}\left(B_{k}\right)\right)<\infty$, then $\mu_{p}\left(B_{k}\right)<\infty$ and $a_{p}\left(E_{B_{k}}\right)=0$ since $a_{p} \in S\left(M_{0}, \mu_{p}\right)$. Thus

$$
\begin{aligned}
a_{p}\left(E_{B_{k}}\right)- & J^{\mu_{p}}\left(f_{p}^{-1}\left(\ell_{p}^{k-1}\right)^{-1}\left(B_{k}\right), g_{p}^{-1}\left(B_{k}\right)\right) \\
& =-\left(\mu_{p}\left(f_{p}^{-1}\left(\ell_{p}^{k-1}\right)^{-1}\left(B_{k}\right)\right)-\mu_{p}\left(g_{p}^{-1}\left(B_{k}\right)\right)\right)>-\sigma_{p}^{k-1}\left(B_{k}\right) .
\end{aligned}
$$

If $\sigma_{p}^{k-1}\left(N_{k+1}\right)=\mu_{p}\left(f_{p}^{-1}\left(\ell_{p}^{k-1}\right)^{-1}\left(N_{k+1}\right)\right)<\infty$, then $\mu_{p}\left(N_{k+1}\right)<\infty$ and $a_{p}\left(E_{B_{j}}\right)=0(j=k+1, \ldots, m)$. Since

$$
\begin{gathered}
\sum_{j=1}^{m} a\left(E_{B_{j}}\right)=a\left(E_{A}\right)=J^{\mu}\left(f^{-1}(A), g^{-1}(A)\right), \quad A=\left(\ell^{k-1}\right)^{-1}(A), \\
a\left(E_{B_{j}}\right)=J^{\mu}\left(f^{-1}\left(\ell^{k-1}\right)^{-1}\left(B_{j}\right), g^{-1}\left(B_{j}\right)\right) \quad(j=1, \ldots, k-1),
\end{gathered}
$$

it follows that

$$
\begin{aligned}
& a_{p}\left(E_{B_{k}}\right)=\sum_{j=1}^{m} a_{p}\left(E_{B_{j}}\right)-\left(\sum_{j=1}^{k-1} a_{p}\left(E_{B_{j}}\right)+\sum_{j=k+1}^{m} a_{p}\left(E_{B_{j}}\right)\right) \\
&= J^{\mu_{p}}\left(f_{p}^{-1}\left(\ell_{p}^{k-1}\right)^{-1}(A), g_{p}^{-1}(A)\right)-\sum_{j=1}^{k-1} J^{\mu_{p}}\left(f_{p}^{-1}\left(\ell_{p}^{k-1}\right)^{-1}\left(B_{j}\right), g_{p}^{-1}\left(B_{j}\right)\right) \\
&= J^{\mu_{p}}\left(f_{p}^{-1}\left(\ell_{p}^{k-1}\right)^{-1}\left(N_{k}\right), g_{p}^{-1}\left(N_{k}\right)\right) \\
& a_{p}\left(E_{B_{k}}\right)-J^{\mu_{p}}\left(f_{p}^{-1}\left(\ell_{p}^{k-1}\right)^{-1}\left(B_{k}\right), g_{p}^{-1}\left(B_{k}\right)\right) \\
&=J^{\mu_{p}}\left(f_{p}^{-1}\left(\ell_{p}^{k-1}\right)^{-1}\left(N_{k+1}\right), g_{p}^{-1}\left(N_{k+1}\right)\right) \\
&=\mu_{p}\left(f_{p}^{-1}\left(\ell_{p}^{k-1}\right)^{-1}\left(N_{k+1}\right)\right)-\mu_{p}\left(g_{p}^{-1}\left(N_{k+1}\right)\right)<\sigma_{p}^{k-1}\left(N_{k+1}\right)
\end{aligned}
$$


The continuity of the function $t=t(p)$ follows from the continuity of $a_{p}\left(E_{B_{k}}\right), J^{\mu_{p}}\left(f_{p}^{-1}\left(\ell_{p}^{k-1}\right)^{-1}\left(B_{k}\right), g_{p}^{-1}\left(B_{k}\right)\right)$ in $p$ and $J^{\sigma_{p}^{k-1}}\left(\varphi_{t}\left(B_{k}\right), B_{k}\right)$ in $(p, t)$ (cf. Lemma 3.2).

These observations justify the definition of the map $\ell_{k}$, and it is readily seen to satisfy the required conditions. This completes the inductive step and we obtain the map $\ell^{m}$.

This map satisfies the conditions on $\ell$ except $(1)^{\prime}$. On the $n$-disk $A \cap L$ we compare the two maps $\left.\sigma^{m}\right|_{A \cap L},\left.\tau\right|_{A \cap L}: P \rightarrow \mathcal{M}_{\mathrm{g}}^{\partial}\left(A \cap L,\left.m\right|_{A \cap L}\right.$-reg $)$, where

$$
\sigma^{m}=\ell_{*}^{m}\left(\left.\left(f_{*} \mu\right)\right|_{A}\right), \quad \tau=g_{*} \mu: P \rightarrow \mathcal{M}_{\mathrm{g}}^{\partial}\left(A,\left.m\right|_{A^{-}} \text {reg }\right) .
$$

Since

$$
\begin{aligned}
& \sigma^{m}(A \cap L)-\tau(A \cap L)=J^{\mu}\left(f^{-1}\left(\ell^{m}\right)^{-1}(A \cap L), g^{-1}(A \cap L)\right) \\
& \quad=J^{\mu}\left(f^{-1}\left(\ell^{m}\right)^{-1}(A), g^{-1}(A)\right)-\sum_{k=1}^{m} J^{\mu}\left(f^{-1}\left(\ell^{m}\right)^{-1}\left(B_{k}\right), g^{-1}\left(B_{k}\right)\right) \\
& \quad=a\left(E_{A}\right)-\sum_{k=1}^{m} a\left(E_{B_{k}}\right)=0,
\end{aligned}
$$

Theorem 2.1 yields a map

$$
\xi: P \rightarrow \mathcal{H}_{\partial}\left(A \cap L,\left.m\right|_{A \cap L} \text {-reg }\right)_{1} \cong \mathcal{H}_{\partial \cup(A-L)}\left(A,\left.m\right|_{\left.A^{-r e g}\right)_{1}}\right.
$$

such that $\left.\left(\xi_{*} \sigma^{m}\right)\right|_{A \cap L}=\left.\tau\right|_{A \cap L}$ and $\xi_{p}=\mathrm{id}_{A}$ if $\left.\sigma_{p}\right|_{A \cap L}=\left.\tau_{p}\right|_{A \cap L}$. Finally, the composition $\ell=\xi \ell^{m}$ satisfies all of the required conditions, and this completes the proof. (We note that since the maps $\left.\varphi_{t}\right|_{N_{k}}(t \in(-1,1))$ have locally common compact support, the map $h$ also has locally common compact support.)

Let $L^{0}=\emptyset$ and $f^{0} \equiv \operatorname{id}_{M_{0}}, g^{0} \equiv \operatorname{id}_{M_{0}}$.

Lemma 4.2. There exists a sequence $\left(K_{k}, L_{k}, f^{k}, g^{k}\right)(k=1,2, \ldots)$ which satisfies the following conditions:

$\left(1_{k}\right) K_{k}, L_{k} \in \mathcal{D}\left(M_{0}\right)$ and $L_{k-1} \subset \operatorname{Int}_{M_{0}} K_{k}, K_{k} \subset \operatorname{Int}_{M_{0}} L_{k}$.

$\left(2_{k}\right) \quad$ (i) $f^{k}, g^{k}: P \rightarrow \mathcal{H}_{\partial}^{\mathrm{c}}\left(M_{0}, m_{0} \text {-reg }\right)_{1}^{*}$ are continuous maps,

(ii) $f^{k}=\varphi^{k} f^{k-1}$ and $g^{k}=\psi^{k} g^{k-1}$ for some continuous maps $\varphi^{k}$ : $P \rightarrow \mathcal{H}_{\partial \cup L_{k-1}}^{\mathrm{c}}\left(M_{0}, m_{0}-\mathrm{reg}\right)_{1}^{*}$ and $\psi^{k}: P \rightarrow \mathcal{H}_{\partial \cup K_{k}}^{\mathrm{c}}\left(M_{0}, m_{0} \text {-reg }\right)_{1}^{*}$.

$\left(3_{k}\right) \quad$ (i) $\operatorname{diam} A \leq 2^{-k}, \operatorname{diam}\left(g_{p}^{k-1}\right)^{-1}(A) \leq 2^{-k}\left(A \in \mathcal{C}\left(\mathrm{cl}_{M_{0}}\left(M_{0}-K_{k}\right)\right)\right)$,

(ii) $\operatorname{diam} B \leq 2^{-k}, \operatorname{diam}\left(f_{p}^{k}\right)^{-1}(B) \leq 2^{-k}\left(B \in \mathcal{C}\left(\operatorname{cl}_{M_{0}}\left(M_{0}-L_{k}\right)\right)\right)$.

$\left(4_{k}\right) \quad$ (i) $f_{*}^{k} \mu=g_{*}^{k-1} \mu$ on $K_{k}$ and $g_{*}^{k} \mu=f_{*}^{k} \mu$ on $L_{k}$,

(ii) $J^{\mu}\left(\left(f^{k}\right)^{-1}(A),\left(g^{k-1}\right)^{-1}(A)\right)=a\left(E_{A}\right)\left(A \in \mathcal{C}\left(\operatorname{cl}_{M_{0}}\left(M_{0}-K_{k}\right)\right)\right)$, (iii) $J^{\mu}\left(\left(f^{k}\right)^{-1}(B),\left(g^{k}\right)^{-1}(B)\right)=a\left(E_{B}\right)\left(B \in \mathcal{C}\left(\mathrm{cl}_{M_{0}}\left(M_{0}-L_{k}\right)\right)\right)$. 
$\left(5_{k}\right) \quad$ (i) $\left\{\left(f_{p}^{k}\right)^{-1}\right\}_{p}$ is equicontinuous on $\mathrm{cl}_{M_{0}}\left(M_{0}-K_{k}\right)$ with respect to $\left.d\right|_{M_{0}}$.

(ii) $\left\{\left(g_{p}^{k}\right)^{-1}\right\}_{p}$ is equicontinuous on $\mathrm{cl}_{M_{0}}\left(M_{0}-L_{k}\right)$ with respect to $\left.d\right|_{M_{0}}$.

$\left(6_{k}\right)$ If $p \in P$ and $a_{p}=0$, then $f_{p}^{k}=g_{p}^{k}=\operatorname{id}_{M_{0}}$.

Proof. Suppose we have constructed $\left(K_{k-1}, L_{k-1}, f^{k-1}, g^{k-1}\right)$.

Since $\left\{\left(g_{p}^{k-1}\right)^{-1}\right\}_{p}$ is equicontinuous on $\mathrm{cl}_{M_{0}}\left(M_{0}-L_{k-1}\right)$, we can find $K_{k} \in \mathcal{D}\left(M_{0}\right)$ which satisfies $\left(1_{k}\right)$ and $\left(3_{k}\right)$. By applying Lemma 4.1 to the data $\left(L_{k-1}, K_{k}, f^{k-1}, g^{k-1}, \mu, a\right)$, we obtain $\varphi_{k}$ and $f^{k}$ which satisfy $\left(2_{k}\right)$, $\left(4_{k}\right)-\left(6_{k}\right)$.

Since $\left\{\left(f_{p}^{k}\right)^{-1}\right\}_{p}$ is equicontinuous on $\operatorname{cl}_{M_{0}}\left(M_{0}-K_{k}\right)$, we can find $L_{k} \in \mathcal{D}\left(M_{0}\right)$ which satisfies $\left(1_{k}\right)$ and $\left(3_{k}\right)$. By applying Lemma 4.1 to the data $\left(K_{k}, L_{k}, g^{k-1}, f^{k}, \mu,-a\right)$, we obtain $\psi^{k}$ and $g^{k}$ which satisfy $\left(2_{k}\right)$, $\left(4_{k}\right)-\left(6_{k}\right)$. This completes the inductive step.

Lemma 4.3. Suppose $\left(K_{k}, L_{k}, f^{k}, g^{k}\right)(k=1,2, \ldots)$ is the sequence of Lemma 4.2.

(1) The maps $f^{k}: P \rightarrow \mathcal{H}_{\partial}\left(M_{0}, m_{0} \text {-reg }\right)_{1}(k=1,2, \ldots)$ converge $\left.d\right|_{M_{0} \text { - }}$ uniformly to a continuous map $f: P \rightarrow \mathcal{H}_{\partial}\left(M_{0}, m_{0} \text {-reg }\right)_{1}$.

(2) The maps $g^{k}: P \rightarrow \mathcal{H}_{\partial}\left(M_{0}, m_{0} \text {-reg }\right)_{1}(k=1,2, \ldots)$ converge $\left.d\right|_{M_{0}-}$ uniformly to a continuous map $g: P \rightarrow \mathcal{H}_{\partial}\left(M_{0}, m_{0}-\mathrm{reg}\right)_{1}$.

(3) $\left.f^{-1}\right|_{L_{k}}=\left.\left(f^{k}\right)^{-1}\right|_{L_{k}}$ and $\left.g^{-1}\right|_{K_{k}}=\left.\left(g^{k-1}\right)^{-1}\right|_{K_{k}}(k=1,2, \ldots)$,

(4) $f_{*} \mu=g_{*} \mu$,

(5) If $p \in P$ and $a_{p}=0$, then $f_{p}=g_{p}=\mathrm{id}_{M_{0}}$.

Proof. This follows from the same argument as in [3, Proof of Lemma 4.8].

Proof of Theorem 1.2' . We show that the continuous map $h=g^{-1} f$ : $P \rightarrow \mathcal{H}_{\partial}\left(M_{0}, m_{0} \text {-reg }\right)_{1}, h_{p}=g_{p}^{-1} f_{p}$, satisfies the required conditions.

(1) By Lemma 4.3(4) we have $h_{*} \mu=\mu$ and from Lemma 2.2 it follows that

$$
h_{p} \in \mathcal{H}_{\partial}\left(M_{0}, \mu_{p} \text {-reg }\right)_{1} \cap \mathcal{H}_{\partial}\left(M_{0}, \mu_{p}\right)=\mathcal{H}_{\partial}\left(M_{0}, \mu_{p}\right)_{1} .
$$

(2) For each $F \in \mathcal{Q}\left(E_{M_{0}}\right)$ there exist $k \geq 1$ and $A_{1}, \ldots, A_{m} \in$ $\mathcal{C}\left(\operatorname{cl}_{M_{0}}\left(M_{0}-K_{k}\right)\right)\left(A_{i} \neq A_{j}(i \neq j)\right)$ such that $F=E_{A_{1}} \cup \cdots \cup E_{A_{m}}$ (disjoint). Thus, it suffices to show that $c_{h_{p}}^{\mu_{p}}\left(E_{A}\right)=a_{p}\left(E_{A}\right)$ for all $k \geq 1$ and $A \in \mathcal{C}\left(\mathrm{cl}_{M_{0}}\left(M_{0}-K_{k}\right)\right)$.

Since $f_{p}^{-1} \in \mathcal{H}_{E_{M_{0}}}\left(M_{0}\right)$, we have $E_{f_{p}^{-1}(A)}=E_{A}$. Since $\left.f_{p}^{-1}\right|_{K_{k}}=\left.\left(f_{p}^{k}\right)^{-1}\right|_{K_{k}}$ and $\left.g_{p}^{-1}\right|_{K_{k}}=\left.\left(g_{p}^{k-1}\right)^{-1}\right|_{K_{k}}$ (Lemma 4.3(3)), we have

$$
f_{p}^{-1}(A)=\left(f_{p}^{k}\right)^{-1}(A) \quad \text { and } \quad g_{p}^{-1}(A)=\left(g_{p}^{k-1}\right)^{-1}(A) .
$$


Then from Lemma $4.2\left(4_{k}\right)$ it follows that

$$
\begin{aligned}
c_{h_{p}}^{\mu_{p}}\left(E_{A}\right) & =c_{h_{p}}^{\mu_{p}}\left(E_{f_{p}^{-1}(A)}\right)=J^{\mu_{p}}\left(f_{p}^{-1}(A), h_{p} f_{p}^{-1}(A)\right)=J^{\mu_{p}}\left(f_{p}^{-1}(A), g_{p}^{-1}(A)\right) \\
& =J^{\mu_{p}}\left(\left(f_{p}^{k}\right)^{-1}(A),\left(g_{p}^{k-1}\right)^{-1}(A)\right)=a_{p}\left(E_{A}\right) .
\end{aligned}
$$

(3) Lemma 4.3(5) yields $h_{p}=\operatorname{id}_{M_{0}}$.

5. Proof of Theorem $\mathbf{1 . 2}$ in the general case. In this final section we prove Theorem 1.2 in the general case. According to the usual strategy (cf. [5]), the mapping theorem in $[4,3]$ is used to reduce the noncompact $n$-manifold case to the case of $n$-cube with ends (Theorem $1.2^{\prime}$ ). The correspondence between these cases under the proper map given by the mapping theorem has been discussed in Section 3.3.

Throughout this section, $M^{n}$ is a noncompact connected $n$-manifold and $\omega \in \mathcal{M}_{\mathrm{g}}^{\partial}(M)$.

Lemma 5.1 ([3, Proposition 4.2, Proof of Theorem 4.1 (p. 252)]). There exists a compact 0-dimensional subset $E \subset \partial I^{n}\left(E \subset I_{1}\right.$ if $\left.n \geq 2\right)$ and a continuous proper surjection $\pi: I^{n}-E \rightarrow M$ which satisfies the following conditions:

(i) $U \equiv \pi\left(\operatorname{Int} I^{n}\right)$ is a dense open subset of Int $M$ and the restriction $\left.\pi\right|_{\text {Int } I^{n}}:$ Int $I^{n} \rightarrow U$ is a homeomorphism.

(ii) $F \equiv \pi\left(\partial I^{n}-E\right)=M-U$ and $\omega(F)=0$.

(iii) The induced map $\bar{\pi}: E \rightarrow E_{M}$ is a homeomorphism.

(iv) The induced measure $\pi^{*} \omega$ is $\left.m\right|_{I^{n}-E}$-regular.

Let $M_{0}=I^{n}-E$ and $m_{0}=\left.m\right|_{M_{0}}$. We have $\omega_{0} \equiv \pi^{*} \omega \in \mathcal{M}_{g}^{\partial}\left(M_{0}, m_{0}\right.$-reg $)$.

Proof of Theorem 1.2. By the considerations in Section 3.3 the map $\pi$ in Lemma 5.1 induces the reciprocal homeomorphisms and the commutative diagram of three squares:

$$
\begin{array}{cccccc}
\mathcal{M}_{\mathrm{g}}^{\partial}\left(M_{0}, m_{0} \text {-reg }\right) \underset{\pi^{*}}{\stackrel{\pi_{*}}{\rightleftarrows}} \mathcal{M}_{\mathrm{g}}^{\partial}(M, \omega \text {-reg }), & \pi^{*}=\left(\pi_{*}\right)^{-1}, \\
\mathcal{H}_{\partial}\left(M_{0}, m_{0} \text {-reg }\right)_{1} & \supset & \mathcal{H}_{\partial}\left(M_{0}, \pi^{*} \mu_{p}\right)_{1} & \stackrel{c^{\pi^{*} \mu_{p}}}{\longrightarrow} & \mathcal{S}\left(M_{0}, \pi^{*} \mu_{p}\right) & \subset \mathcal{S}\left(E_{M_{0}}\right) \\
\pi_{*} \downarrow & \pi_{*} \downarrow & \cong \downarrow \bar{\pi}_{*} & \cong \downarrow \bar{\pi}_{*} \\
\mathcal{H}_{F}(M, \omega \text {-reg })_{1} & \supset & \mathcal{H}_{F}\left(M, \mu_{p}\right)_{1} & \stackrel{c^{\mu_{p}}}{\longrightarrow} & \mathcal{S}\left(M, \mu_{p}\right) & \subset \mathcal{S}\left(E_{M}\right)
\end{array}
$$

The maps $\mu$ and $a$ admit lifts to $M_{0}$ :

$$
\pi^{*} \mu: P \rightarrow \mathcal{M}_{\mathrm{g}}^{\partial}\left(M_{0}, m_{0} \text {-reg }\right) \quad \text { and } \quad \tilde{a}=\left(\bar{\pi}_{*}\right)^{-1} a: P \rightarrow \mathcal{S}\left(E_{M_{0}}\right) .
$$

Since $a_{p} \in S\left(M, \mu_{p}\right)$, the third square in the above diagram implies that $\widetilde{a}_{p} \in \mathcal{S}\left(M_{0}, \pi^{*} \mu_{p}\right)$. Theorem $1.2^{\prime}$ provides a continuous map $\widetilde{h}: P \rightarrow$ 
$\mathcal{H}_{\partial}\left(M_{0}, \pi^{*} \omega \text {-reg }\right)_{1}$ such that for each $p \in P$,

$(1)^{\prime} \widetilde{h}_{p} \in \mathcal{H}_{\partial}\left(M_{0}, \pi^{*} \mu_{p}\right)_{1}$,

$(2)^{\prime} c_{\widetilde{h}_{p}}^{\pi^{*} \mu_{p}}=\widetilde{a}_{p}$

$(3)^{\prime}$ if $\widetilde{a}_{p}=0$, then $\widetilde{h}_{p}=\mathrm{id}_{M_{0}}$.

We show that the map

$$
h=\pi_{*} \widetilde{h}: P \rightarrow \mathcal{H}_{F}(M, \omega-\text { reg })_{1} \subset \mathcal{H}_{\partial}(M, \omega \text {-reg })_{1}
$$

satisfies the required conditions.

(1) The condition (1)' and the first square imply that $h_{p} \in \mathcal{H}_{F}\left(M, \mu_{p}\right)_{1}$ $\subset \mathcal{H}_{\partial}\left(M, \mu_{p}\right)_{1}$.

(2) From $(2)^{\prime}$ and the second square it follows that

$$
c_{h_{p}}^{\mu_{p}}=c^{\mu_{p}} \pi_{*}\left(\widetilde{h}_{p}\right)=\bar{\pi}_{*} c^{\pi^{*} \mu_{p}}\left(\widetilde{h}_{p}\right)=\bar{\pi}_{*}\left(c_{\widetilde{h}_{p}}^{\pi^{*} \mu_{p}}\right)=\bar{\pi}_{*}\left(\widetilde{a}_{p}\right)=a_{p} .
$$

(3) If $a_{p}=0$, then $\widetilde{a}_{p}=0$ and $\widetilde{h}_{p}=\operatorname{id}_{M_{0}}$. This implies that $h_{p}=\operatorname{id}_{M}$. This completes the proof.

Proof of Theorem 1.1. The required section is obtained by applying Theorem 1.2 to the data: $P=\mathcal{S}(M, \omega), \mu \equiv \omega$ and $a$ is the inclusion $\mathcal{S}(M, \omega) \subset \mathcal{S}\left(E_{M}\right)$.

Suppose $\mathcal{G}$ is any subgroup of $\mathcal{H}_{E_{M}}(M, \omega)$ with $\mathcal{H}_{\partial}(M, \omega)_{1} \subset \mathcal{G}$. Consider the restriction $\left.c^{\omega}\right|_{\mathcal{G}}: \mathcal{G} \rightarrow \mathcal{S}(M, \omega)$.

COROllary 5.1.

(1) $\left(\mathcal{G},\left.\operatorname{Ker} c^{\omega}\right|_{\mathcal{G}}\right) \cong\left(\left.\operatorname{Ker} c^{\omega}\right|_{\mathcal{G}}\right) \times(\mathcal{S}(M, \omega), 0)$.

(2) $\left.\operatorname{Ker} c^{\omega}\right|_{\mathcal{G}}$ is a strong deformation retract of $\mathcal{G}$.

Proof. (1) The required homeomorphism is defined by

$$
\varphi: \mathcal{G} \rightarrow\left(\left.\operatorname{Ker} c^{\omega}\right|_{\mathcal{G}}\right) \times \mathcal{S}(M, \omega), \quad \varphi(h)=\left(\left(s\left(c_{h}^{\omega}\right)\right)^{-1} h, c_{h}^{\omega}\right) .
$$

The inverse is given by $\varphi^{-1}(f, a)=s(a) f$.

(2) Since the topological vector space $\mathcal{S}(M, \omega)$ admits a strong deformation retraction onto $\{0\}$, the conclusion follows from (1).

\section{References}

[1] S. R. Alpern and V. S. Prasad, Typical Dynamics of Volume-Preserving Homeomorphisms, Cambridge Tracts Math. 139, Cambridge Univ. Press, 2000.

[2] R. Berlanga, A mapping theorem for topological sigma-compact manifolds, Compos. Math. 63 (1987), 209-216.

[3] - , Groups of measure-preserving homeomorphisms as deformation retracts, J. London Math. Soc. (2) 68 (2003), 241-254. 
[4] R. Berlanga and D. B. A. Epstein, Measures on sigma-compact manifolds and their equivalence under homeomorphism, ibid. 27 (1983), 63-74.

[5] M. Brown, A mapping theorem for untriangulated manifolds, in: Topology of 3Manifolds and Related Topics, M. K. Fort (ed.), Prentice-Hall, Englewood Cliffs, 1963, 92-94.

[6] A. Fathi, Structures of the group of homeomorphisms preserving a good measure on a compact manifold, Ann. Sci. École Norm. Sup. (4) 13 (1980), 45-93.

[7] J. Oxtoby and S. Ulam, Measure preserving homeomorphisms and metrical transitivity, Ann. of Math. 42 (1941), 874-920.

[8] T. Yagasaki, Groups of measure-preserving homeomorphisms of noncompact 2-manifolds, Topology Appl. 154 (2007), 1521-1531.

[9] - Groups of volume-preserving diffeomorphisms of noncompact manifolds and mass flow toward ends, preprint.

Division of Mathematics

Faculty of Engineering and Design

Kyoto Institute of Technology

Matsugasaki, Sakyoku, Kyoto 606-8585, Japan

E-mail: yagasaki@kit.ac.jp

Received 17 November 2005;

in revised form 19 November 2007 